Implications for Guideline Developers/Users Guideline developers can use GRADE and these methods when there is no evidence or low/very low quality evidence from RCTs.

\section{ADDRESSING CONTINUOUS DATA FOR PARTICIPANTS EXCLUDED FROM TRIAL ANALYSIS: A GUIDE}

${ }^{1} S$ Ebrahim, ${ }^{2} E$ Akl, ${ }^{5} \mathrm{R}$ Mustafa, ${ }^{3} \mathrm{X}$ Sun, ${ }^{1} \mathrm{~S}$ Walter, ${ }^{1} \mathrm{D}$ Heels-Ansdell, ${ }^{4} \mathrm{P}$ Alonso-Coello, ${ }^{1} \mathrm{~B}$ Johnston, ${ }^{1} \mathrm{G}$ Guyatt. ${ }^{1} \mathrm{M}$ MMaster University, Hamilton, Canada; ${ }^{2}$ American University of Beirut, Beirut, Lebanon; ${ }^{3}$ The Centre for Health Research, Kaiser Permanente Northwest, Portland, USA; ${ }^{4}$ beroamerican Cochrane Centre, CIBERESP-IIB Sant Pau, Barcelona, Spain; ${ }^{5}$ University of Missouri, Kansas City, USA

\section{0:1136/bmjgs-2013-002293.87}

Background Guideline developers addressing quality of evidence commonly confront studies with missing data.

Objectives To develop a framework for assessing risk of bias resulting from missing participant data for continuous outcomes in systematic reviews.

Methods We developed a range of progressively more stringent imputation strategies to challenge the robustness of the pooled estimates. We applied our approach to two systematic reviews.

Results We used 5 sources of data for imputing means for participants with missing data: [A] the best mean score among the intervention arms of included trials, [B] the best mean score among the control arms of included trials, [C] the mean score from the control arm of the same trial, [D] the worst mean score among the intervention arms of included trials, [E] the worst mean score among the control arms of included trials. Using these sources of data, we developed four progressively more stringent imputation strategies. In the first example review, effect estimates were diminished and lost significance as the strategies became more stringent, suggesting the need to rate down confidence in estimates of effect for risk of bias. In the second review, effect estimates maintained statistical significance using even the most stringent strategy, suggesting missing data does not undermine confidence in the results.

Discussion Our approach provides rigorous yet reasonable and relatively simple, quantitative guidance that guideline developers can use for judging the impact of risk of bias as a result of missing participant data in systematic reviews of continuous outcomes.

\section{HANDLING TRIAL PARTICIPANTS WITH MISSING DATA IN META-ANALYSES OF DICHOTOMOUS OUTCOMES: GUIDANCE FOR SYSTEMATIC REVIEWERS}

${ }^{1} \mathrm{E}$ Akl, ${ }^{2,4} \mathrm{~B}$ Johnston, ${ }^{3} \mathrm{P}$ Alonso-Coello, ${ }^{4} \mid$ Neumann, ${ }^{4} \mathrm{~S}$ Ebrahim, ${ }^{5} \mathrm{M}$ Briel, ${ }^{4} \mathrm{D}$ Cook, ${ }^{4} \mathrm{G}$ Guyatt. ${ }^{1}$ American University of Beirut, Beirut, Lebanon; ${ }^{2}$ Sick Kids Research Institute, Toronto, Canada; ${ }^{3}$ Ibero-American Cochrane Centre, Barcelona, Spain; ${ }^{4}$ McMaster University, Hamilton, Ontario; ${ }^{5}$ Basel Institute for Clinical Epidemiology, Basel, Switzerland

\section{0:1136/bmjgs-2013-002293.88}

Background Systematic reviewers including all randomised participants in their meta-analyses need to make assumptions about the outcomes of those with missing data.

Objectives To provide systematic review authors with guidance on dealing with participants with missing data for dichotomous outcomes.
Methods The authors used an iterative process of suggesting guidance and obtaining feedback to arrive at a proposed approach.

Results For participants with missing data, systematic reviewers can use a range of plausible assumptions in the intervention and control arms. Extreme assumptions include 'all' or 'none' of the participants had an event, but these assumptions are not plausible. Less extreme assumptions may draw on the incidence rates within the trial (e.g., same incidence in the trial control arm) or in all trials included in the meta-analysis (e.g., highest incidence among control arms of all included trials). The primary metaanalysis may use either a complete case analysis or a plausible assumption. Sensitivity meta-analyses to test the robustness of the primary meta-analysis results should include extreme plausible assumptions. When the meta-analysis results are robust to extreme plausible assumptions, inferences are strengthened. Vulnerability to extreme plausible assumptions suggests rating down confidence in estimates of effect for risk of bias.

Conclusions This guide proposes an approach to establishing confidence in estimates of effect when systematic reviewers are faced with missing participant data for binary dichotomous outcomes in randomised trials.

\section{ASSESSMENT OF THE EVIDENCE FOR DIAGNOSTIC TESTS AND STRATEGIES: A SYSTEMATIC REVIEW OF AVAILABLE TOOLS}

${ }^{1,2} \mathrm{R}$ Mustafa, ${ }^{1,6,7} \mathrm{~W}$ Wiercioch, ${ }^{1} \mathrm{M}$ Falavigna, ${ }^{1} \mathrm{Y}$ Zhang, ${ }^{3} \mathrm{~B}$ Prediger, ${ }^{4} \mathrm{~A}$ Cheung, ${ }^{1} \mathrm{~L}$ Ivanova, ${ }^{5}$ I Arevalo-Rodriguez, ${ }^{1} \mathrm{H}$ Schünemann, on behalf of the DU-Diagnosis SR group. ${ }^{1}$ McMaster University, Hamilton, Canada; ${ }^{2}$ University of Missouri-Kansas City, Kansas City, USA; ${ }^{3}$ Germany; ${ }^{4}$ University of British Columbia, Vancouver, Canada; ${ }^{5}$ Universidad Nacional de Colombia, Bogota, Columbia; ${ }^{6}$ Universidade Federal do Rio Grande do Sul, Porto Alegre, Brazil; ${ }^{7}$ National Institute of Science and Technology for Health Technology Assessment, Porto Alegre, Brazil

\section{0:1136/bmjqs-2013-002293.89}

Background The challenges facing guideline developers when making recommendations about diagnostic tests and strategies (DTS) are considerably different when compared to treatment recommendations.

Objectives To identify, describe and compare all available instruments, checklists, critical appraisal tools, and indices designed for assessing the quality of evidence (QoE) or strength of recommendations (SoR) dealing with diagnostic tests and strategies.

Methods We conducted a comprehensive systematic search of the literature including state of the art diagnostic guidelines, methods papers and diagnostic systematic reviews.

Results We identified 45 tools and modifications of existing tools to assess the QoE and SoR of DTS. Most tools acknowledge the importance of assessing the QoE and SoR separately. Most tools include individual quality criteria and study design but no tool rates all quality criteria suggested by the GRADE working group. Only two tools explicitly consider factors that increase the confidence in the evidence. When moving from evidence to recommendations, patient values and preferences and resources were rarely considered.

Discussion There is confusion about the terminology that describes the various factors that influence the QoE and SoR. The criteria for evaluating the QoE and moving from evidence to recommendations are incomplete for most guideline development frameworks that we evaluated.

Implications for Guideline Developers/Users The GRADE approach is the most complete approach encompassing all factors but users will benefit from a better description of the 